\title{
ACCURACY ANALYSIS FOR KOLMOGOROV ENTROPY USED IN STUDYING THE CHAOTIC DYNAMICS OF CFB REACTORS BASED ON SOLIDS CONCENTRATION FLUCTUATIONS
}

\author{
S. V. Manyele \\ Department of Chemical and Process Engineering \\ College of Engineering and Technology \\ University of Dar es Salaam, P.O. Box 35131, Date s Salaam \\ smanyele@.cpe.udsm.ac.tz
}

\begin{abstract}
ABSTARCT
A study on the chaotic dynamics of a high flux circulating fluidized bed (CFB) riser (10-m high and 76-mm i.d.) using the maximum likelihood estimate of the Kolmogorov entropy $\left(K_{M L}\right)$ is reported. The signals used were measured by using a solids concentration fiber optic probe, accuracy of which is reported based on statistical analysis. The sensitivity analysis of the parameters used for computing $K_{M L}$ was conducted to identify optimum settings, based on the standard error, $s\left(K_{M L}\right)$ and the nature of PDF of the $b$-values from the reconstructed phase space trajectories. The length of time series, $N$, sampling frequency, $f_{s}$, and number of embedding dimensions, $D_{i m}$, and $\bar{b}$, strongly affect the accuracy of $K_{M L}$ values. A relationship between $K_{M L}$ and $\bar{b}$ was established, which was obeyed by experimental data from all operating conditions studied, and for all sampling frequencies. The chaotic dynamics of the CFB was studied by examining the effect of increasing the local solids concentration at different axial elevations and different operating conditions in a high flux riser. It was concluded that the $K_{M L}$ method is highly accurate when $N>$ 3,000 data points, for which the effect of $D_{\text {im }}$ is negligible and PDFs of the b-values becomes similar in shape based on $\sigma(b), S_{k}(b)$ and $K_{u}(b)$.
\end{abstract}

Keywords: Solids concentration fluctuations, accuracy of measurements, time series analysis, chaos analysis, Kolmogorov entropy.

\section{INTRODUCTION}

Multiphase reactors exhibit complex non-linear oscillations (mixed periodic and chaotic oscillations). Moreover, the dynamics of such systems exhibit both spatial complexity (different dynamics from one location to another) and sometimes temporal complexity (different dynamics from one time to another) (Addison, 1997), especially due to feed variations and gas pulsations. This complexity has been studied using both one-dimensional approach (statistical and spectral analyses) and also a multi-dimensional approach using phase-space statistics (chaos analysis).

Chaos analysis is not the only tool for the analysis of the complex dynamics of multi-phase reactors; other methods include statistical analysis and spectral analysis. However, statistical analysis (using mean, standard deviation and properties of the PDF) does not keep track of time-dependency behavior of the multiphase reactors (van der Stappen et al., 1993), i.e., even when the signal is arranged in ascending order of values, the same value is obtained. Chaos analysis has been used in the study of weather (Lorentz model), economics, epidemiology and ecology (Schaffer, 1985; Müller et al., 1995), demography, and in medical diagnostics (Hoyer et al., 1998). The analysis of multiphase dynamics using chaos analysis has been so far developed and used for analysis of pressure fluctuations (Manyele, et al., 2002a; 2003), solids concentration signals (Cheng et al., 1998), and temperature fluctuations.

In the analysis of solids concentration signals form a CFB, the mean value of the signal describes what is measured while standard deviation and other statistical measures represent noise and other interferences. In the CFB, such interferences are caused by gas flow pulsations and formation and breakdown of clusters (Manyele et al., 2002b). Different from macroflow, the microflow studies are normally focused on quantifying the fluctuating component of the signal (Manyele et al., 2003); however, the fluctuations must be within reasonable range (Soong et al., 1994).

During solids concentration measurements in the CFB, the final state is regarded to be the steady state condition when all flows remain constant. Starting signal recording some time after the process has started attains the conditions of stationarity. However, the signals must still be tested for stationarity before chaos analysis can be applied (Finney et al., 1996). The stationarity condition is attained when the statistical averages 
(i.e. mean, standard deviation) remain constant along different segments of the signal or time series.

In a CFB riser, for example, dilute and dense phase flow take place dominantly at the center and in the wall region and bottom section, respectively (Johnson and Johnson, 2001; Manyele et al., 2002a). Chaos analysis has been able to distinguish such flows based on strong sensitivity of its parameters (correlation dimension and Kolmogorov entropy) (Bai et al., 1999; Manyele et al., 2002a; 2003; 2006). Similarly, the axial flow structure along the CFB, which varies widely (entrance, acceleration, transition and the exit sections), have been identified using chaos analysis of pressure fluctuations and solids concentration signals from high density risers (Manyele et al., 2006; 2002a; Bai et al., 1999) and downers (Cheng et al., 1998; Manyele et al., 2003).

The Kolmogorov entropy characterizes the sensitivity of the gas-solids flow to small disturbances and its rate of information loss; it is also a measure of the predictability of the changes in the gas-solids flow (Grassbeger and Procaccia, 1983; Daw, 1990; 1991; 1992). Higher entropy signifies higher rate of information loss (or lower predictability) and also stronger dependency on small disturbances. Recently, $K_{M L}$ have been extensively used to study the chaotic behavior of CFB risers and downers based on both solids concentration data (Cheng et al., 1998; Manyele et al., 2003; 2006).

The most acceptable algorithm for computation of Kolmogorov entropy is the maximum likelihood method developed by Schoulten et al. (1994), denoted as $K_{M L}$. However, the accuracy of the data processing technique for determination of $K_{M L}$ depends on a number of parameters such as sampling frequency, length of the time series, time average solids concentration, and number of embedding dimensions used in phase space reconstruction. This paper analyzes the effect of these parameters using solids concentration data from CFBs. After establishing the optimum values for these parameters, the chaotic behavior of the CFB was studied by examining the effect of local solids concentration in a high flux riser. Moreover, the accuracy of the measurement technique for solids concentration fluctuations was analyzed using statistical methods.

\section{EXPERIMENTAL DETAILS}

The signals used in this study were measured in two different units: (a) one of the risers of a 10-m CFB twin-riser system, with 76-mm i.d. operated at high flux, and (b) from a downer reactor 100-mm diameter and $9.3 \mathrm{~m}$ tall, operated at low flux conditions. The solids circulating in each of these systems were spent FCC catalyst with a mean diameter of $67 \mu \mathrm{m}$ and a particle density of 1500 $\mathrm{kg} / \mathrm{m}^{3}$. Figure 1 shows the setup of the two units. The riser was operated at high flux conditions $\left(U_{g}=\right.$ 5.5 to $10.0 \mathrm{~m} / \mathrm{s}$ and $G_{s}=100-550 \mathrm{~kg} / \mathrm{m}^{2} \mathrm{~s}$, while the downer was operated under dilute conditions $\left(U_{g}=3.7\right.$ to $10.0 \mathrm{~m} / \mathrm{s}$ and $G_{s}=50$ to $200 \mathrm{~kg} / \mathrm{m}^{2} \mathrm{~s}$ ). The details of the two units have been reported by Manyele et al. (2003; 2006).

Solids concentration fluctuations measurements were conducted using a reflective-type optical fiber concentration-probe for both units. The active area in the probe tip was approximately $2 \mathrm{~mm} \times 2 \mathrm{~mm}$, consisting of approximately 8000 emitting and receiving quartz fibers, each having a diameter of $15 \mu \mathrm{m}$. More details of this probe including its calibration procedure can be found from Zhang et al. (1998). Measurements were taken on several axial levels and at several radial positions. The sampling time was 30 seconds at a frequency of $970 \mathrm{~Hz}$. Each stored signal consisted of 27,000 data points. Data analysis was conducted using FORTAN codes. 


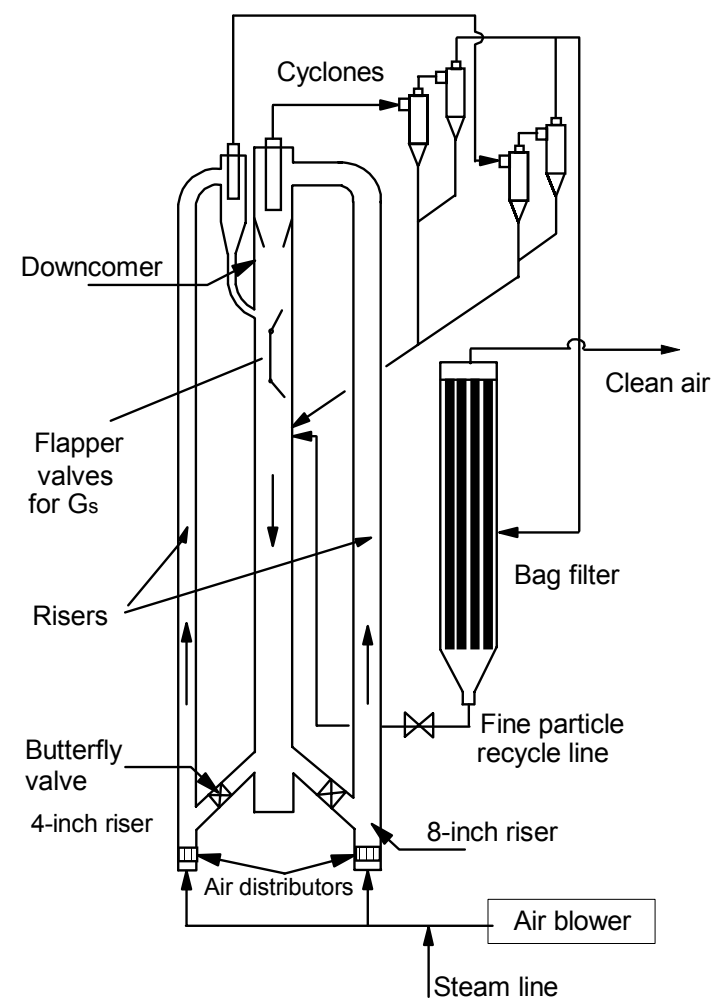

Riser

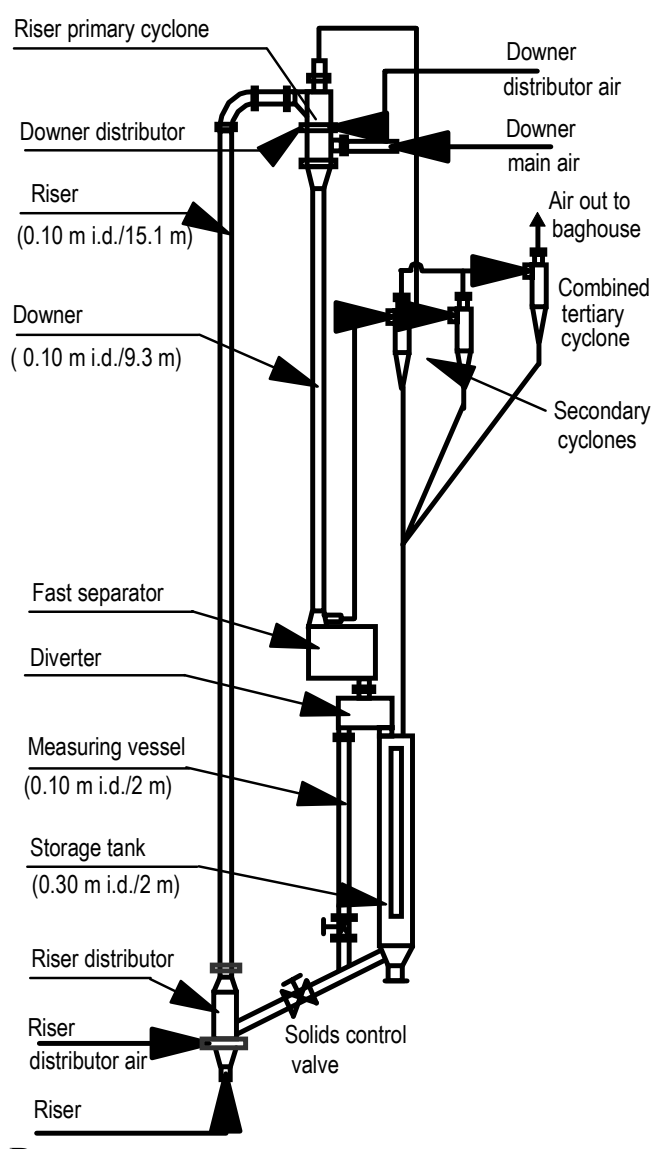

Downer

Figure 1: Experimental setup for downer and riser CFBs

\section{DETERMINATION OF KOLMOGOROV ENTROPY USING MAXIMUM LIKELIHOOD METHOD}

The maximum likelihood approach was proposed for the estimation of Kolmogorov entropy of experimental data, $K_{\mathrm{ML}}$ (Schoulten et al., 1994). Because the computations of the Kolmogorov entropy using the method of Grassberger and Proccaccia (1983) do not have a known standard error, the maximum likelihood method has gained acceptance in the multiphase reactor signal processing. However, this method needs analysis before it can be implemented. The accuracy of the computational results for $K_{\mathrm{ML}}$ depends on the following factors: the average value of $b$, the sampling frequency, the effect of the length of the time series used in the computation, and number of embedding dimensions used.

This maximum likelihood method is based on the average number of steps before the exponential divergence between reconstructed trajectories exceeds the average absolute deviation, $A A D$, of the original time series, using a time delay of one sampling interval, $\square t$, for phase space reconstruction, as shown in equation (1).

$$
K_{M L}=-\frac{1}{\tau} \ln \left(1-\frac{1}{\bar{b}}\right)
$$

where $\bar{b}$ is the average number of steps before the distance between two points exceeds the $A A D$ or the crossing distance, and $\tau=\Delta t$. A positive value of $K_{\mathrm{ML}}$ is a necessary condition for a system to be chaotic.

The average absolute deviation $(A A D)$ is simply the average value of the departure of the instantaneous values of the solids concentration from the mean value (Schoulten and van den Bleek, 1998; Marzochella et al., 1997; van der Stappen et al., 1993b), expressed mathematically as per equation (2):

$$
A A D=\frac{1}{N} \sum_{i=1}^{N}\left(\left|X_{i}-\bar{X}\right|\right)
$$

where $N$ is number of data points in the signal and $X$ is the one-dimensional series of solids concentration values. 


\section{RESULTS AND DISCUSSION}

\section{Accuracy of the Measurement System}

The accuracy of measurements was expressed in terms of the coefficient of variation, CV (the ratio of the standard deviation to the mean), and signal to noise ratio, SNR (the ratio of the mean value to the standard deviation). A plot showing CV and SNR versus the time-averaged solids concentration, $\varepsilon_{\mathrm{s}}$, for a wide range of operating conditions is shown in Figure 2 (using data from a downer reactor).

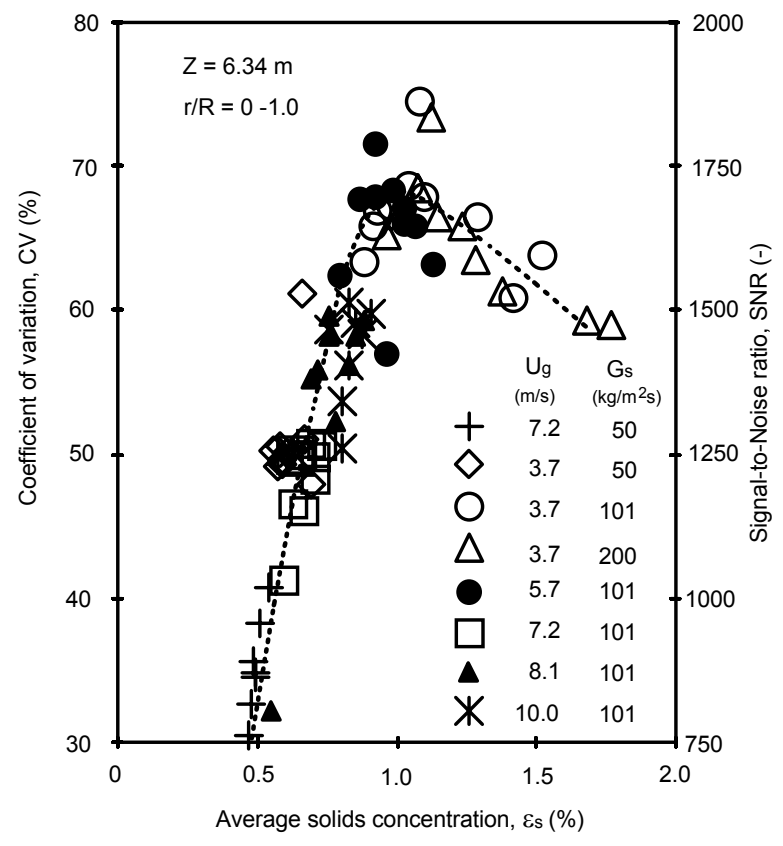

Figure 2: Variation of the coefficient of variation (CV) and signal-to-noise ratio (SNR) with the average solids concentration in the downer reactor (sample data from the fully developed section)

For $\varepsilon_{s}$ less than $1.0 \%, C V$ increases faster reaching a maximum at $\varepsilon_{s} \approx 1.0 \%$, before decreasing again as $\varepsilon_{S}$ approaches $2.0 \%$. The $C V$ varied between 30 $70 \%$ showing that the mean value is always higher than the fluctuations. The corresponding values of $S N R$ range from $750-1750$, which are sufficiently higher signifying that the noise component is smaller. Moreover, the variation of $U_{g}$ and $G_{s}$ has no significant difference on $C V$ and $S N R$.

\section{Accuracy of Skewness and Kurtosis used to analyze the Properties of PDFs}

In this study, Skewness and Kurtosis were employed in analyzing the properties of the PDF of sampled solids concentration signals and also for the PDF of the counted number of points in phase space during computation of $K_{\mathrm{ML}}$. High positive Skewness indicates that the PDF have a long tail towards higher values (to the right), while the Kurtosis measures the tendency of the PDF to have a high peak.

The accuracy of both $S_{k}$ and $K_{u}$ depend strongly on the number of samples used in computation, that is $N$. For the calculated values of Skewness and Kurtosis to be meaningful, the corresponding standard deviation as the estimators of both $S_{k}$ and $K_{u}$ of the underlying distribution, must be known.

For the ideal case of a normal (Gaussian) distribution, the standard deviation of the computed $S_{k}$ as an estimator of the Skewness is approximately $S_{k, N}=\sqrt{15 / N}$, which is equal to 0.024 for $N=$ 27,000 used in this study. The accuracy of the computed Skewness is high when the computed value is many times as large as $S_{k, N}$. To examine the accuracy of the computed values of Skewness of the sampled signals, the values are plotted versus the time-averaged solids concentration, $\varepsilon_{s}$, as shown in Figure 3. The horizontal dashed line indicates a base line for $S_{k, N}=0.024$. The computed values of Skewness ranged from 1 to 10 , which are clearly higher than 0.024 , about $40-100$ times, for all operating conditions. The Skewness also shows a maximum at $\varepsilon_{s}=1.0 \%$, similar to $C V$. 


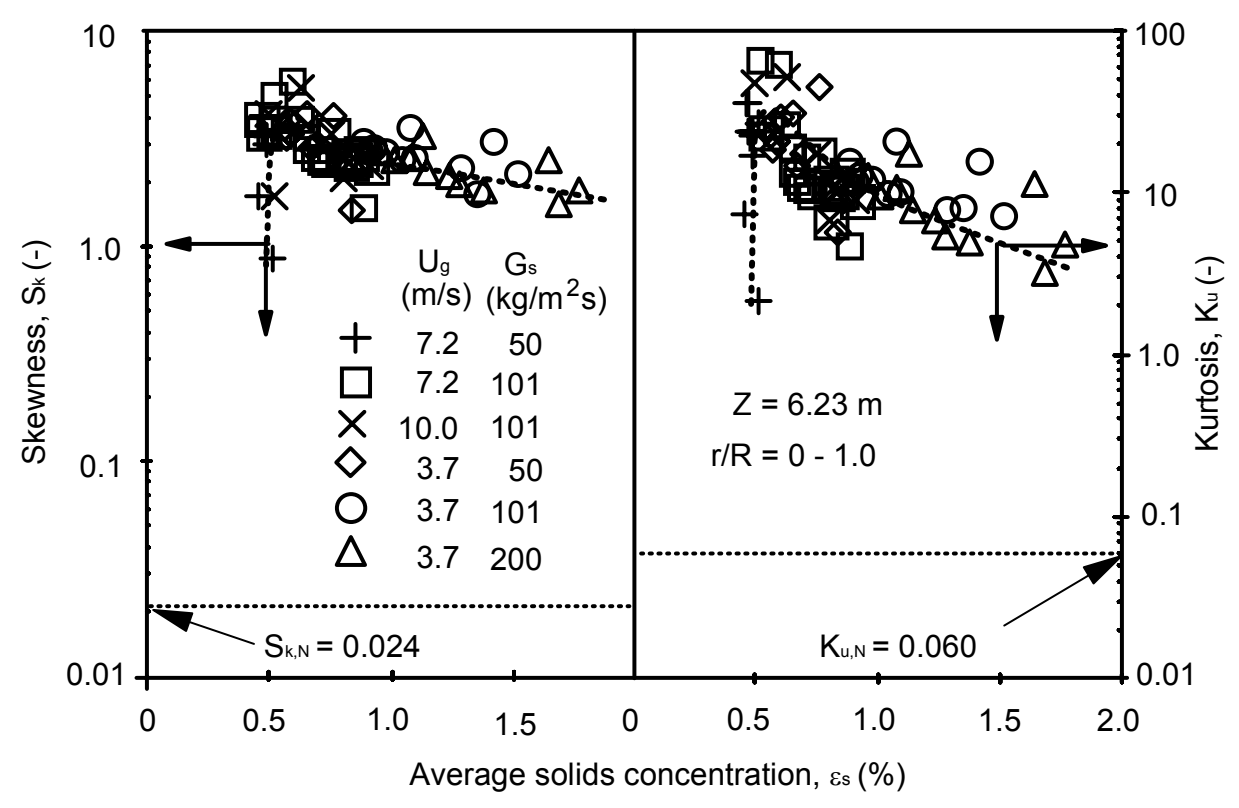

Figure 3: Range of values for both Skewness and Kurtosis in the downer reactor (sample data from the fully developed section).

Based on the normal (Gaussian) distribution, again the standard deviation of the Kurtosis as an estimator of the Kurtosis of the underlying distribution is given by $K_{u, N}=\sqrt{96 / N}$. The same approach was used as for Skewness. The accuracy was inferred by comparing the computed values of $K_{u}$ with the $K_{u, N}$. Figure 3 shows also the variation of $K_{u}$ with $\varepsilon_{s}$ for similar operating conditions. The $K_{u}$ values range from 1 to 100 and are far higher than the $K_{u, N}=0.060$. Similarly, the Kurtosis shows a peak as $\mathcal{E}_{s}$ is increased from 0.5 to $2.0 \%$. The above analysis was then used to examine the PDFs of the b-values created from the reconstructed trajectories, with $N=10^{6}$.

\section{The effect of $\bar{b}$ of the Reconstructed Vector on the $K_{M L}$}

Figure 4 shows the range of $b$-values for signal reconstructed using 100 embedding dimensions for $N=5000$ data points. The determination of $b$ values requires counting of points on the attractor before the separation distance from a given arbitrary stationary point exceeds $A A D$. Once $A A D$ is exceeded, the number of points counted is recorded. The computer is then instructed to jump to another arbitrary point on the attractor and the counting is repeated.

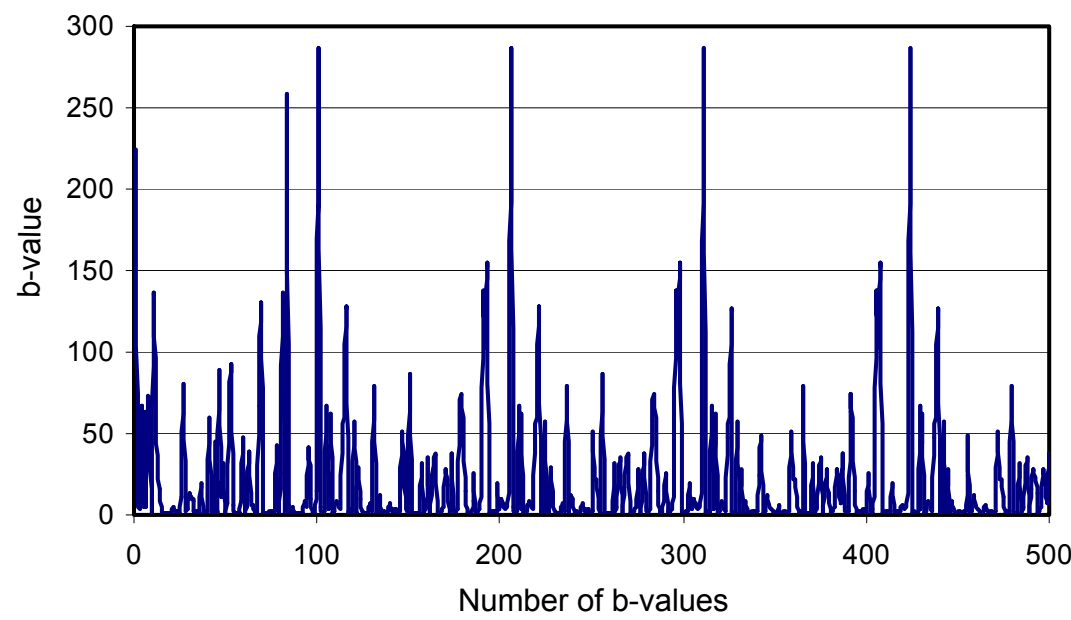

Figure 4: Range of $b$-values for a solids concentration signal embedded at $D_{i m}=100$ and $N=5,000$ using a signal from a high flux riser $\left(U_{g}=8.0 \mathrm{~m} / \mathrm{s} ; G_{s}=300 \mathrm{~kg} / \mathrm{m}^{2} \mathrm{~s} ; Z=6.34 \mathrm{~m}\right.$ and $\left.r / R=0\right)$ 
Using the maximum-likelihood method, several values of $b$ are determined, the average of which is used to calculate the $K_{\mathrm{ML}}$. With a large value of $M$, it is also interesting to examine the nature of the PDF. The sequence of values of $b$ obtained from a given time series were used to study the probability distribution function (PDF). This was studied by computing the standard deviation and Skewness of the b-values as $N$ changes, as shown in Figure 5. For $N<1,000$ points, the effect of $D_{i m}$ is stronger leading to totally different shapes of PDFs for the b-values whenever $D_{\text {im }}$ is changed, as observed from the wide variation in the standard deviation, $\sigma(b)$, Skewness, $S_{k}(b)$ and Kurtosis, $K_{u}(b)$. Meanwhile, the Skewness increases with $N$ until it levels off for $N>1,000$. With standard deviation between 6.0 and 8.0 (for $N>1,000$ ), it shows that the values of $b$ are relatively similar.

In the analysis of b-values, the Skewness was observed to range between 2.5 to 3.0 at higher $N$. Based on the accuracy analysis results from Figure 3 , the experimental values are far higher than the $S_{k, N}$, signifying that the computed $S_{k}$ values of the underlying PDF of $b$-values is highly accurate. The fact that the Skewness values for the PDF of $b$ values are far higher than the $S_{k, N}$ for the PDF of a normal distribution, i.e. 0.004 for $N=10^{6}$ counts, signifies that the computed Skewness values are of high accuracy.

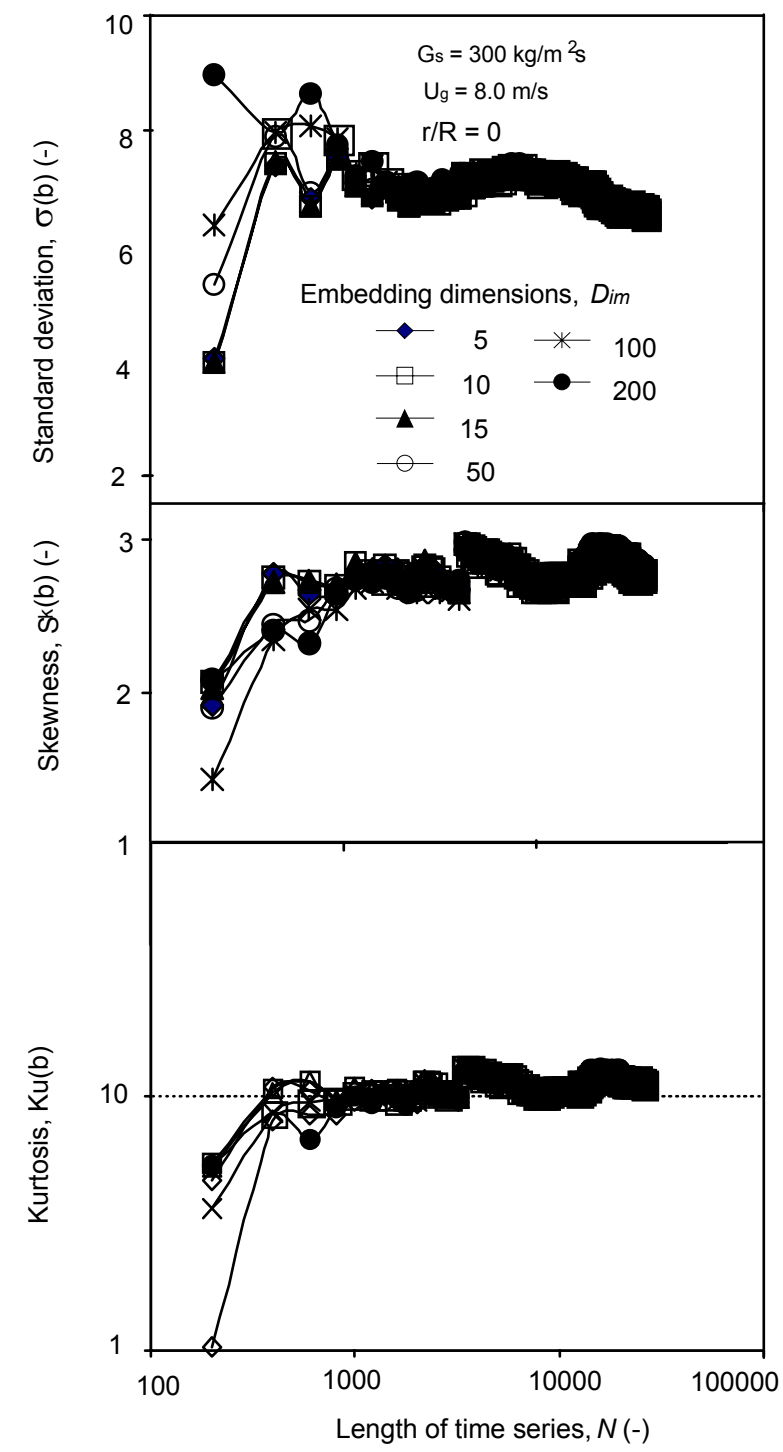

Figure 5: Effect of the time series length, $N$, on the nature of the PDF of $b$-values at different $\mathrm{D}_{\text {im }}$ 


\section{Effect of Sampling Frequency on $K_{M L}$}

The fiber optic probe used for measurements of solids concentration was sampled at a frequency of $970 \mathrm{~Hz}$. With such a fast sampling software and hardware combination, it was possible to reduce the frequency from 970 to $61 \mathrm{~Hz}$, and study the effect of the sampling frequency. However, for accuracy, the highest frequency is preferred because it captures the dynamics to the maximum possible state. Figure 6 shows the effect of $b$ value on the range of values of $K_{\mathrm{ML}}$ at different sampling frequencies. By changing the sampling frequency of the signal a different loci of the $K_{\mathrm{ML}}$ values is obtained.

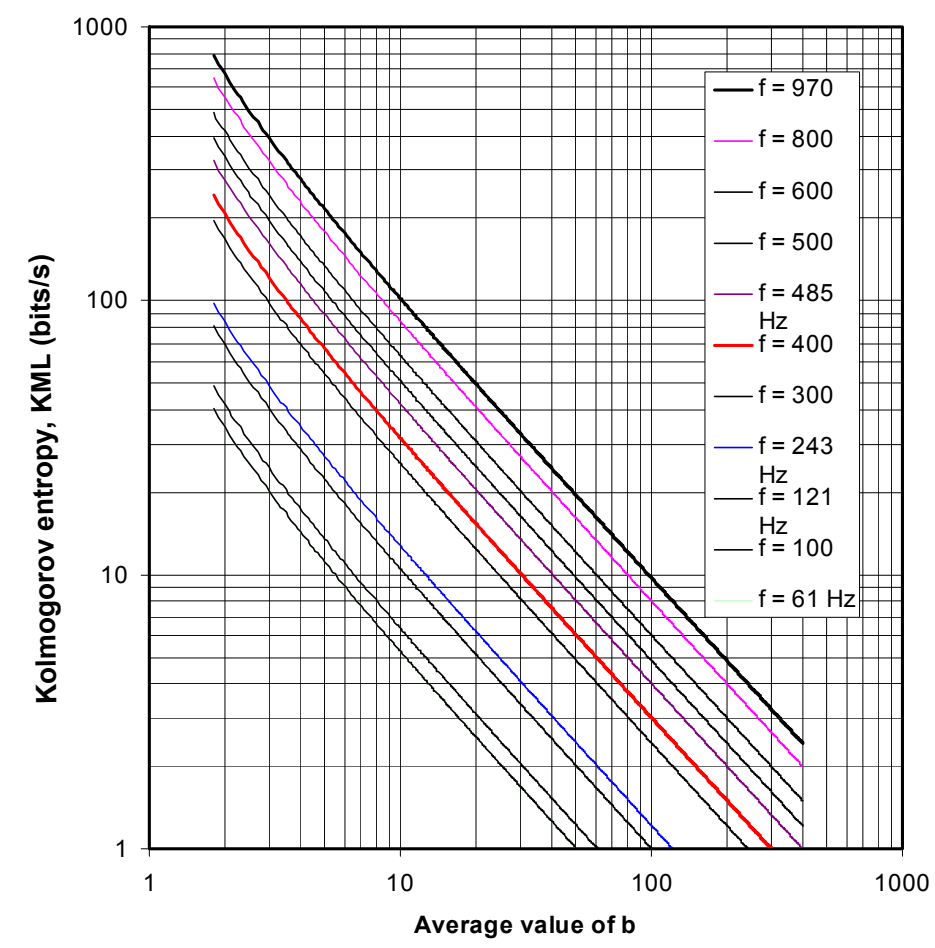

Figure 6: Variation of $K_{M L}$ with the average b-value at different sampling frequencies for signals from a high flux riser $\left(U_{g}=8.0 \mathrm{~m} / \mathrm{s} ; G_{s}=300 \mathrm{~kg} / \mathrm{m}^{2} \mathrm{~s} ; Z=6.34 \mathrm{~m}\right.$ and $\left.r / R=0\right)$

Figure 6 shows that the higher the frequency the higher the $K_{\mathrm{ML}}$ value. Thus, the same system sampled at a lower frequency will lead to lower values of $K_{\mathrm{ML}}$ and vice versa. However, because the locus gives a straight line on a log-log plot, the profiles in the CFB will be the same if the same equations are used. Figure 6 shows also that the higher the average value of $b$ the lower the $K_{\mathrm{ML}}$ value. This is in accordance to the fact that since $\bar{b}$ gives the number of steps before the distance on the attractor exceeds $A A D$, then, lower value of $\bar{b}$ implies that the attractor is more sparsely populated by vector points, or the points are far apart, an indication of a large phase space, and that only a few points are encountered, before $A A D$ is exceeded, implying a higher value of $K_{M L}$.
Figure 7 shows the variation of $K_{\mathrm{ML}}$ with $\bar{b}$ for signals sampled from the high flux riser $t$ different operating conditions. A log-log plot gives a straight line for all conditions, which was fitted by a relationship:

$$
K_{M L} \propto b^{-1.033}
$$

For all operating conditions studied, the relationship presented in equation (3) was observed. The experimental data follows this relationship for dilute and high density conditions, as $G_{\mathrm{s}}$ was changed from 100 to $550 \mathrm{~kg} / \mathrm{m}^{2} \mathrm{~s}$ under the same gas velocity $(8.0 \mathrm{~m} / \mathrm{s})$. Also, the change in velocity from 5.5 to $10.0 \mathrm{~m} / \mathrm{s}$ at constant $G_{\mathrm{s}}$ of $300 \mathrm{~kg} / \mathrm{m}^{2} \mathrm{~s}$ did not affect the relationship. As shown in Figure 6 , a similar relationship will be obtained for all sampling frequencies. 


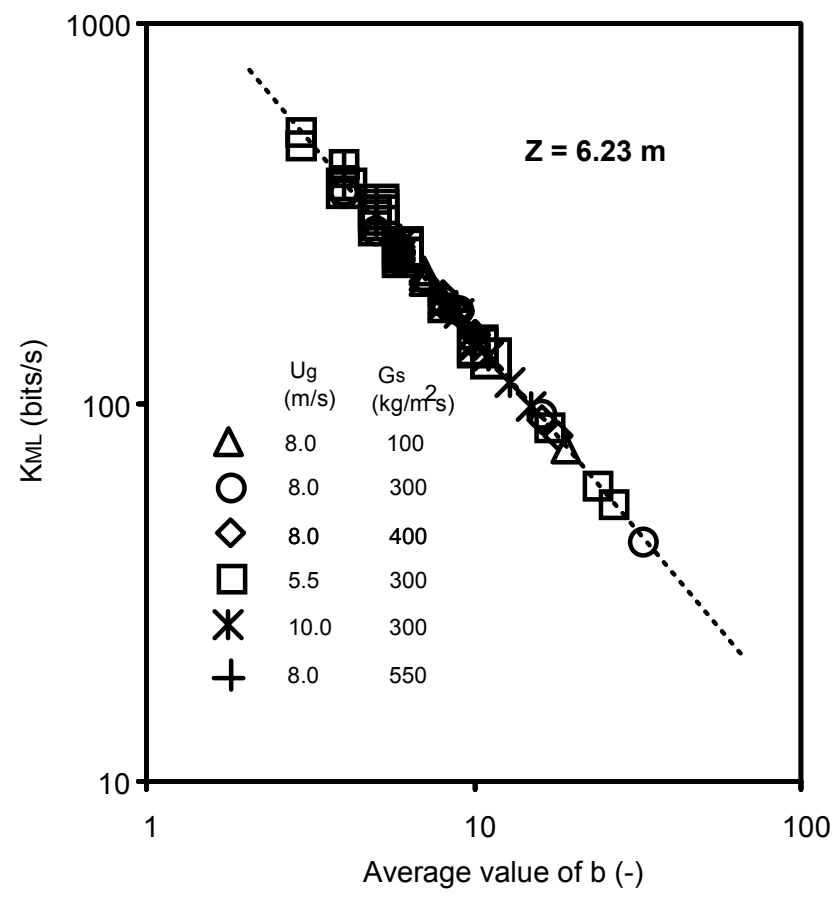

Figure 7: Variation of $K_{M L}$ with $b$ for different operating conditions in a high flux riser in the fully developed section

\section{The effect of Time Series Length on the Actual value and Accuracy of $K_{M L}$}

The length of the time series used in phase space reconstruction is determined by computation speed of the computer. To minimize the computation time, optimization can be done on the computer code or by changing the length of the time series, $N$. Figure 8 shows the semi-log plot of the variation of $K_{M L}$ with $N(200-27,000)$ for a wide range of embedding dimensions $\left(D_{\text {im }}=5-200\right)$ for a signal from a high-density riser.

For $N<1000$ points, the $K_{M L}$ varies widely with $N$ without a clear pattern. However, for $N>1,000$ points, all the values follow a single curve for all $D_{i m}$. This shows that:

(a) The effect of $D_{i m}$ is strong for shorter time series than 1,000 pints. (b) The accuracy and repeatability of $K_{M L}$ values is higher for longer time series.

(c) For longer time series, any number of embedding dimensions will lead to the same range of $K_{M L}$ values.

Schoulten et al. (1994) proposed the standard error for the computed values of $K_{M L}$ to be $M^{-0.5}$, where $M$ is the number of times the distances exceed the average absolute deviation of the signal (number of crossings, which is equal to the number of bvalues). In this study, the optimization of the computation time was based on $N$ for which the accuracy is reported as the former increases. Parallel to the computation of $K_{M L}$, the standard error values were also estimated. Figure 9 shows the log-log plot of the variation of the standard error of $\mathrm{K}_{M L}$ with the length of the time series used for the phase space reconstruction. 


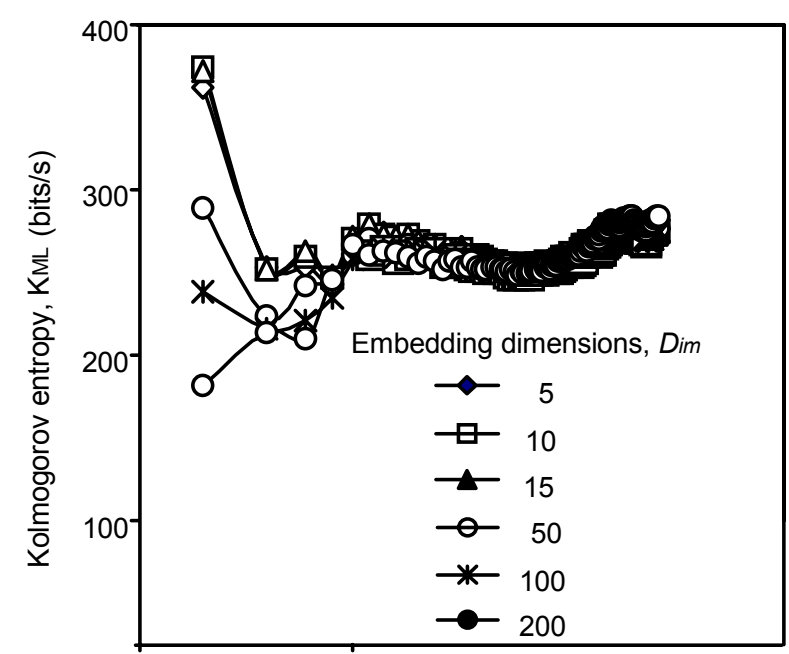

Figure 8: Effect of the time-series length on the $K_{M L}$ values and the corresponding accuracy (based on the standard error values, signal from a high-density riser)

Below $N=3,000$, the standard error in the estimate of $K_{M L}$ is higher and decreases until $N=3,000$, beyond which it remains constant at about 0.001 . The same values of the standard error were obtained regardless of the number of embedding dimension. However, because $s\left(K_{M L}\right)$ decreases for lower values of $N$, the best range of time series length is $N>3,000$ points for higher accuracy. However, the number of embedding dimensions has no effect on the standard error. Therefore, to minimize computer time, a smaller $D_{i m}=5$ and $N$ slightly above 3,000 can be used to estimate $K_{M L}$ values.

It is important to determine the relative standard error of the $K_{\mathrm{ML}}$ (entropy estimate), $\mathrm{s}\left(K_{\mathrm{ML}}\right)$. It is suggested that $s\left(K_{\mathrm{ML}}\right) \leq 0.1 \%$, i.e., $K_{\mathrm{ML}}$ values should be based on at least a sample size the order of $10^{6}$ values of $b$. This counting is performed for a large number of times, about $10^{6}$. The possibility of having such higher number of $b$ values is based on the fact that the embedding dimensions leads to a length of the embedded signal of length $=(N)^{\text {Dim }}$.

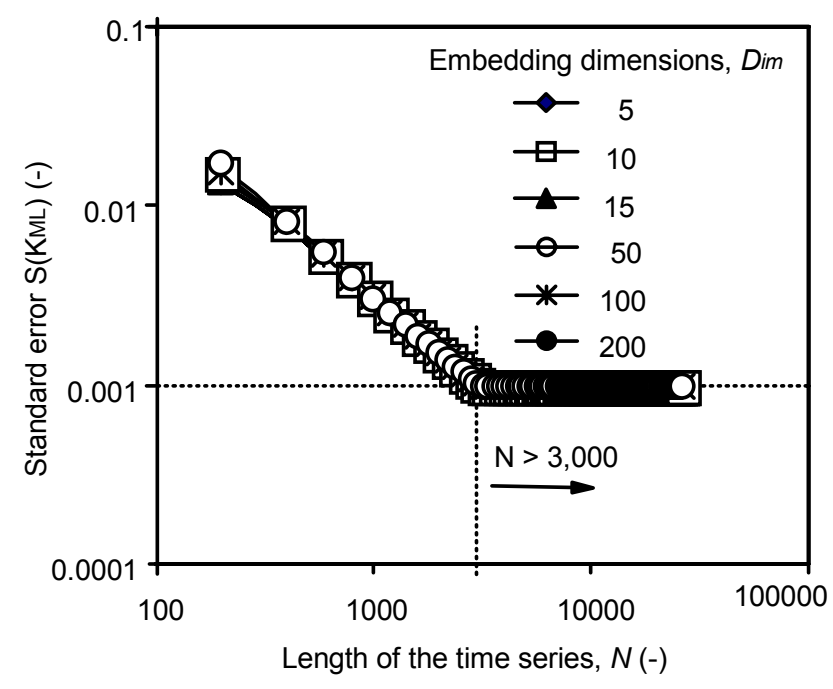

Figure 9: Effect of the time-series length on the accuracy of $K_{M L}$ values (based on the standard error values), signal from a high-density riser

A semi-log plot of $M^{-0.5}$ versus $K_{M L}$ is given in Figure 10. In this case, the number of data points was fixed, and then several values of $K_{M L}$ were determined for each signal, from which the values of $M$ (i.e. the number of crossings or the number of $b$ values) were also computed. Throughout the 
downer, the values of $M^{-0.5}$ ranged only between $0.10 \%$ and $0.25 \%$ for all operating conditions. This range is sufficiently small for experimental signals. Moreover, the higher $K_{M L}$ were determined at higher accuracy than the lower values. Compared to
Figure 6, it implies that higher sampling frequencies from multiphase reactors lead to higher $K_{M L}$ values and also to higher accuracy in the computation of $K_{M L}$.

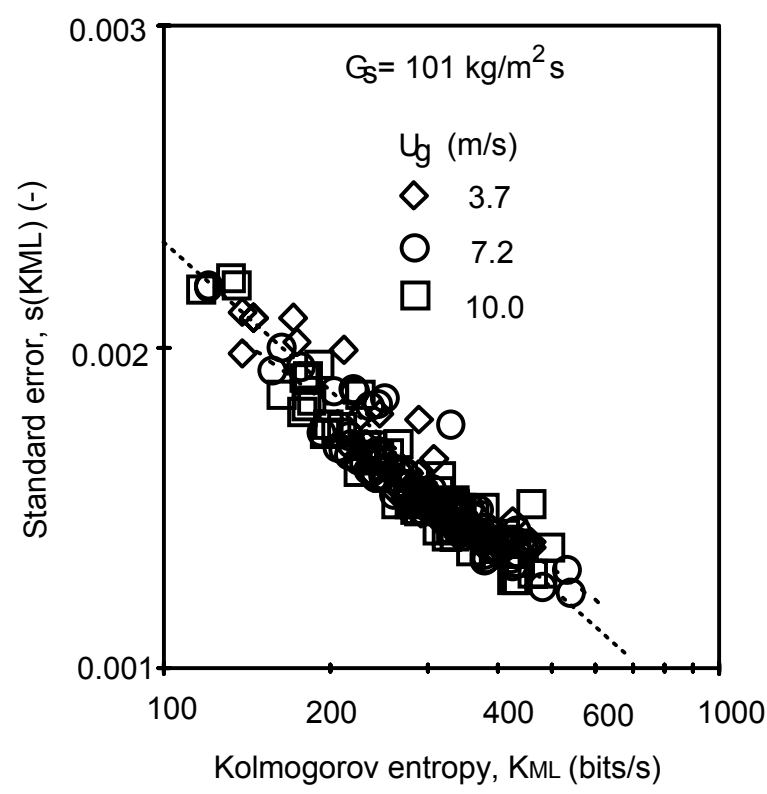

Figure 10: Standard error for the estimated values of entropy, $s\left(K_{\mathrm{ML}}\right)$, for signals from downer reactor operated at different gas velocities

The Effect of Jump-length for restarting a cont of $b$-values on a reconstructed vector

The number of points to be jumped, $J_{\mathrm{m}}$, while looking for an arbitrary point to re-start counting the value of $b$, was not specified by Schoulten $e t$ al. (1994). How $J_{\mathrm{m}}$ affects $K_{\mathrm{ML}}, b$ and the standard error for estimation of $K_{\mathrm{ML}}$ is subject to analysis for experimental data, which has not been done using experimental data from multiphase reactors. Figure 11 shows the effect of increasing $J_{\mathrm{m}}$ on $K_{\mathrm{ML}}$ for different number of embedding dimensions, $D_{i m}$.
The effect of $J_{\mathrm{m}}$ on $K_{M L}$ strongly depends on the number of embedding dimensions, $D_{i m}$. At higher $D_{i m}$, lower values of $K_{M L}$ were observed. Thus, changing $J_{\mathrm{m}}$ changes the value of $K_{M L}$. For experimental data from multiphase reactors, it is recommended to fix the value of $J_{\mathrm{m}}$ throughout the analysis, preferably in the range of $150-350$, when the $K_{M L}$ curves become closer to each other. Further analysis revealed that $J_{\mathrm{m}}$ have no effect on $\bar{b}$, standard deviation, $\sigma(b)$, Skewness, $S_{\mathrm{k}}(b)$, and Kurtosis $K_{\mathrm{u}}(\mathrm{b})$, even when $D_{\mathrm{im}}$ was changed from 5 to 200 . 


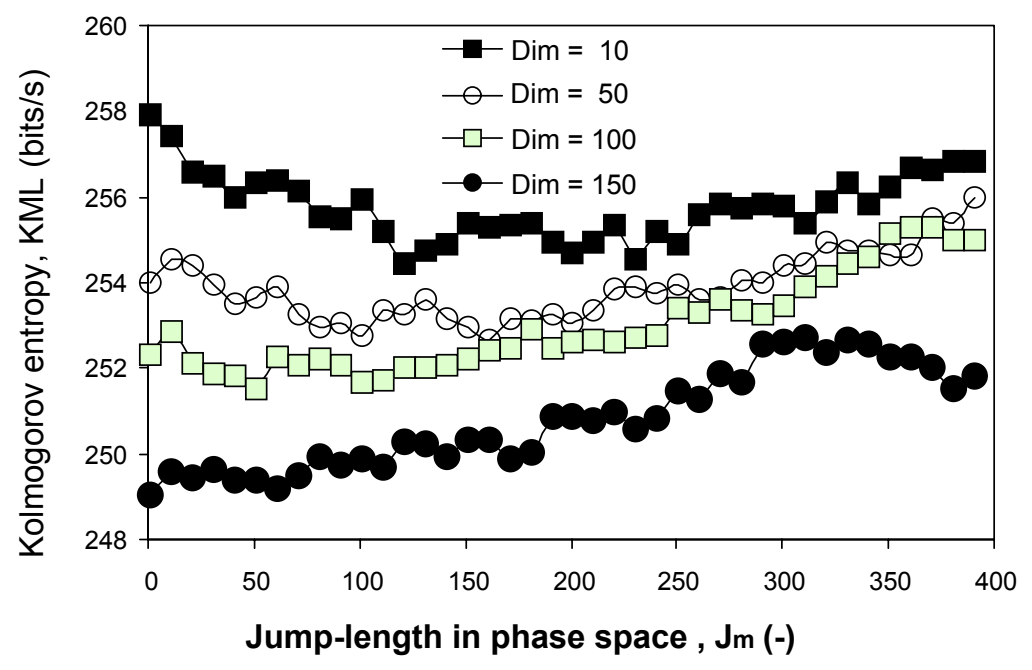

Figure 11: The effect of changing $J_{\mathrm{m}}$ on $K_{\mathrm{ML}}$

\section{Analysis of the Chaotic Behavior of the CFB using $K_{\mathrm{ML}}$}

After setting all the computational parameters to the optimum values $\left(N, f\right.$, and $\left.J_{m}\right)$, according to the results of this analysis, the chaotic analysis of the CFB was investigated, based on the radial profiles, axial profiles and the effect of local solids concentration at different operating conditions. Figure 12 shows the effect of increasing $\varepsilon_{S}$ on both $K_{M L}$ in the fully developed-flow section $(Z=6.34)$ for a wide range of operating conditions in a high flux riser.

Increasing $\varepsilon_{s}$ generally lowers $K_{\mathrm{ML}}$ for all operating conditions. Similar observation can be deduced from Figure 12, when $G_{\mathrm{s}}$ is increased from 300 to $550 \mathrm{~kg} / \mathrm{m}^{2} \mathrm{~s}$ at constant gas velocity of $8.0 \mathrm{~m} / \mathrm{s}$. However, at constant $G_{\mathrm{s}}$, the variation of $K_{\mathrm{ML}}$ with $U_{\mathrm{g}}$ is not clear. Using pressure fluctuations, Manyele et al. (2002a) reported the decrease of $K_{\mathrm{ML}}$ with $G_{\mathrm{s}}$ at constant $U_{\mathrm{g}}$ and also a decrease of $K_{\mathrm{ML}}$ as the apparent solids concentration were increased for all operating conditions. However, the effect of $U_{\mathrm{g}}$ was not elucidated.

The decrease in $K_{\mathrm{ML}}$ as $G_{\mathrm{s}}$ or $\varepsilon_{\mathrm{s}}$ is increased can be attributed to the increased cluster existence time and a decrease in cluster frequency at higher $\varepsilon_{\mathrm{s}}$ (Manyele et al., 2002; Soong et al., 1995; 1994) leading to extended time scales for changes in $\varepsilon_{\mathrm{s}}$ with time, such that the time scales predicted by $K_{\mathrm{ML}}$ becomes longer and hence lower $K_{\mathrm{ML}}$ values are observed. Longer time scales in the signal imply higher b-values and hence lower $K_{\mathrm{ML}}$. On the reconstructed attractor, the number of points counted before the separation distance exceeds $A A D$ becomes higher. In other words, the gas-solids flow stays longer in one dynamic state before it changes into another. Such a system is said to be easily predictable (relatively), attains low rate of information loss and it looses sensitivity to the disturbances.

The effect of increasing $\varepsilon_{\mathrm{s}}$ on $K_{\mathrm{ML}}$ was also studied at different axial elevations in a high flux riser. Figure 13 shows the variation of $K_{\mathrm{ML}}$ with $\varepsilon_{\mathrm{s}}$ at different axial elevations. Along the axial direction, the time scales are different due to several factors. At the bottom, due to the distributor effect (strong gas jets) and solids recirculation, $K_{\mathrm{ML}}$ is higher even at higher $\varepsilon_{\mathrm{s}}$, as shown in Figure 13, which is in accordance to the fact that faster changes take place in the gas-solids flow in this section. In such a section the gas-solids flow is not easily predictable. 


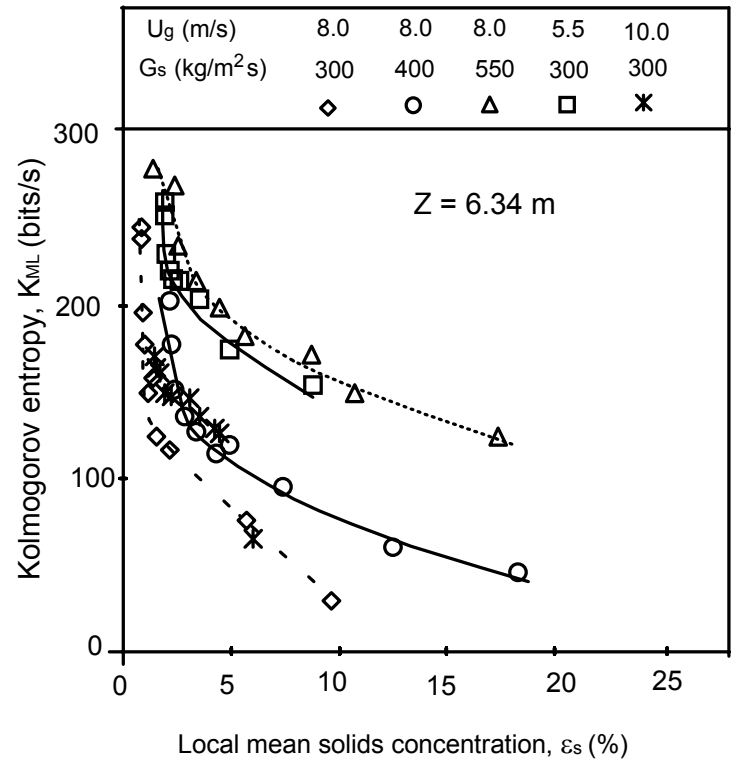

Figure 12: Variation of Kolmogorov entropy with local time-averaged solids concentration in the fully developed section of a high flux $\operatorname{riser}(Z=6.34 \mathrm{~m})$

In the fully developed section, $Z=6.34$ and $8.74 \mathrm{~m}$, there is minimal wall effect, no solids acceleration, such that the time scales are longer than in the bottom. This leads to longer average cycle times (Manyele et al., 2002b) and hence lower $K_{\mathrm{ML}}$ values at comparable values of $\varepsilon_{\mathrm{s}}$.

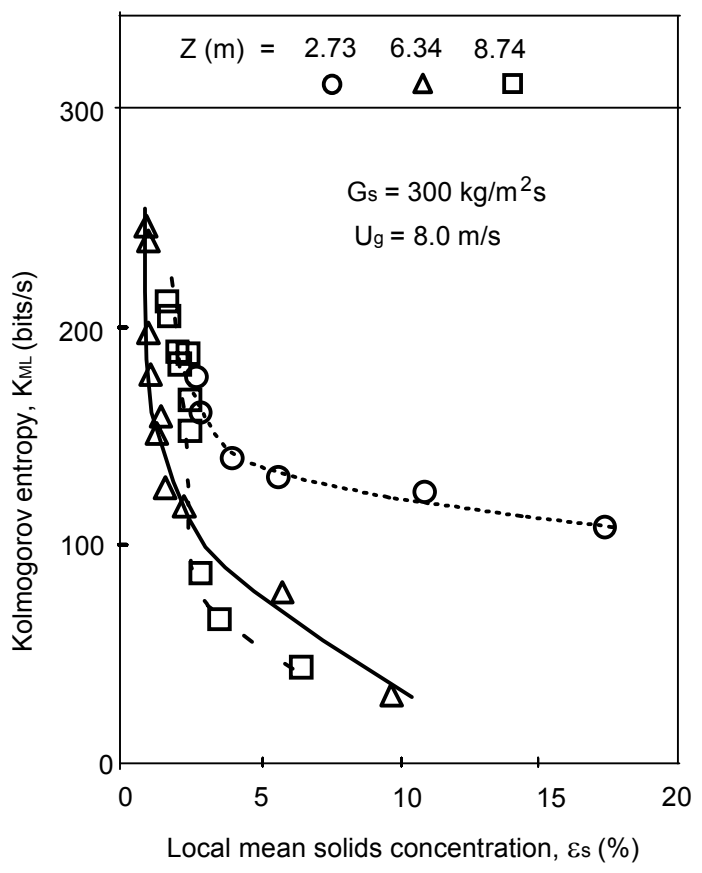

Figure 13: Variation of Kolmogorov entropy with local time-averaged solids concentration in different sections of a high flux riser

\section{CONCLUSIONS}

It can be concluded from this study that:

1) The measurements of solids concentration using a fiber optic probe are highly accurate with SNR of about 750 to 2000 and $C V$ ranging from 30 to $80 \%$.

2) The Skewness and Kurtosis of the solids concentration signals revealed that the properties of the PDF are accurately determined for the signals sampled at a frequency of $970 \mathrm{~Hz}$ and a duration of 30 seconds, which gives $N=27,000$, and $S_{\mathrm{k}, \mathrm{N}}$ and $K_{\mathrm{u}, \mathrm{N}}$ well above 0.024 and 0.060 , respectively, for a normal distribution.

3) The Skewness and Kurtosis of $b$-values ranged between 2.0 to 3.0 and 8.0 to 11.0 , respectively, being well above the $S_{\mathrm{k}, \mathrm{N}}$ and $K_{\mathrm{u}, \mathrm{N}}$ for a normal distribution (using $N=10^{6}$ ).

4) The $K_{\mathrm{ML}}$ is strongly affected by the sampling frequency of the signal from the multiphase reactor, which leads to different values reported in the literature for CFBs. Higher frequency leads to higher values of $K_{\mathrm{ML}}$ and vice versa.

5) The values of $K_{\mathrm{ML}}$ strongly depend on the length of the time series, $N$. For $\mathrm{N}<1,000$ points, the $K_{\mathrm{ML}}$ values depends strongly on the number of embedding dimension, Dim, while the latter was observed to have no effect for $N>1,000$ points.

6) The standard error, $s\left(K_{\mathrm{ML}}\right)$, decreases to a constant minimum at 0.001 , for $N>3,000$ points. The effect of $D_{\mathrm{im}}$ is negligible on the $s\left(K_{\mathrm{ML}}\right)$. Higher values of $K_{\mathrm{ML}}$ are accurately determined than lower values, whereby $s\left(K_{\mathrm{ML}}\right)$ decreases with increasing $K_{\mathrm{ML}}$ value.

7) The $K_{\mathrm{ML}}$ decreases with increasing timeaveraged solids concentration in the riser, due to corresponding longer time scales at higher $\varepsilon_{\mathrm{s}}$. This was observed for all operating conditions and for all axial elevations.

8) The $K_{\mathrm{ML}}$ is a powerful tool for studying the dynamics of CFB reactors using chaos analysis. It can be used accurately for $N>$ 3,000 data points. Higher values of $N$ will lead to longer computations time. 


\section{NOMENCLATURE}

$A A D$ Average absolute deviation (-)

$b \quad$ number of steps before the separation distance exceeds AAD

$C V \quad$ Coefficient of variation (\%)

$D_{i m} \quad$ Number of embedding dimensions (-)

$f \quad$ Sampling frequency $(\mathrm{Hz})$

$G_{s} \quad$ Solids flux $\left(\mathrm{kg} / \mathrm{m}^{2} \mathrm{~s}\right)$

$J_{m} \quad$ Number of points jumped to start a new counting of $b$ values (-)

$K_{M L} \quad$ Kolmogorov entropy determined by maximum likelihood method, (bits/s).

$K_{u} \quad$ Kurtosis (-)

$K_{u, N} \quad$ Kurtosis of a normal distribution

$M \quad$ Number of b-values

$N \quad$ Number of data points of original times series used for reconstruction of multidimensional vector (-)

$s\left(K_{M L}\right) \quad$ Standard error of the computed $K_{M L}$

$S_{k} \quad$ Skewness (-)

$S_{k, N} \quad$ Skewness of a normal distribution (-)

SNR Signal-to-noise ratio (-)

$U_{g} \quad$ Superficial gas velocity $(\mathrm{m} / \mathrm{s})$

Greek letters

$\varepsilon_{s} \quad$ Time -averaged solids concentration (\%)

$\Delta \mathrm{t} \quad$ Sampling time interval (s)

\section{REFERENCES}

Addison, P.S., (1997), "Fractals and Chaos: An Illustrated Course", Institute of Physics Publishing (IOP) Ltd., Bristol, pp. 117-190.

Bai, D., Issangya, A.S., and Grace, J.R., (1999), "Characteristics of Gas-Fluidized Beds in Different Flow Regimes", Ind. Eng. Chem. Res., 38, 803-811.

Cheng, Y.; Wei, F.; Lin, Q.; Jin, Y. A., (1998), Comparison of Local Chaotic Behaviors in a Riser and a Downer. In Fluidization IX. Fan, L.-S.; Knowlton, T.M., Eds.; Engineering Foundation, NY, , pp 613-620.

Daw, C.S., and Halow, J.S., (1991), "Characterization of Voidage and Pressure Signals from Fluidized Beds Using Deterministic Chaos Theory", Proc. of $11^{\text {th }}$ Fluidized Bed Combustion (Ed. E.J. Anthony), ASME, Vol. 2, 777-786.

Daw, C.S., and Halow, J.S., (1992), "Modeling Deterministic Chaos in Gas Fluidized Beds", AIChE Symp. Ser. 88 (289), 61-69.
Daw, C.S., Lawkins, W.F., Downing D.J., and Clapp, Jr., N.E., (1990), "Chaotic Characteristics of a Complex Gas -Solids Flow", Phys. Rev. A, 41(2), 1179-1181.

Finney, C.E.A, Kennel, M.B., Daw, C.S., and Halow, J.S., (1996), "Non-Linear Time Series Diagnostics of Fluidization Quality", AIChE Annual Meeting, Paper 188C, November 14,

Fuller, T.A., Flynn, T.J., and Daw, C.S., (1996), "Analysis of Dynamic Boiler Measurements: A Practical Approach", Chem. Eng. J., 64, 179-189.

Grasberger, P., and Procaccia, I., (1983), "Estimation of the Kolmogorov Entropy from a Strange Signal", Physcal Review, A28, 2591-2594.

Hegger, R., Kantz, H. and Screiber, T., (1999), "Practical Implementation of Nonlinear Time Series Methods: The TISEAN Package, Chaos, 9, 413.

Hoyer, D., Bauer, R., Walter B., and Zwiener, U., (1998), "Estimation of Nonlinear Couplings on the Basis of Complexity and Predictability - A New Method Applied to Cardiotherapy Coordination", IEE Transactions on Biomedical Engineering, 45(5), 545-552

Johnson, H., and Johnson, F., (2001), "Measurement of Local Solids Volume Fraction in Fluidized Bed Boilers", Powder Technol., 115, 13-26.

Manyele, S.V., Pärssinen J., and Zhu, J.-X., (2002b), "Characterizing Particle Aggregates in a Riser Operated at High Solids Flux", Chemical Engineering J., 88, 151-161.

Manyele, S.V., Zhu, J.-X, Khayat, R.E., and Pärssinen, J., (2006), "Analysis of the Chaotic Dynamics of a High-Flux CFB Riser using Solids Concentration", China Particuology, Vol. 88 Nos. 3-4, 136-146

Manyele, S.V., Zhu, J.-X., and Zhang, H., "Analysis of the Microscopic Flow Structure of a CFB Downer Reactor Using Solids Concentration Signal", International Journal of Chemical Reactor Engineering, Volume 1, 
Article A55, Produced by The Berkeley Electronic Press, 2003, pp. 1-17,

Manyele, S.V., Khayat, R.E., and Zhu. J., (2002a), "Investigation of the Dynamics of a High-flux CFB Riser Using Chaos Analysis of Pressure Fluctuations". Chem. Eng. Technology, 25, 1-10.

Marzochella, A., Zijeveld, R.C., Schoulten, J.C., and van den Bleek, C.M., (1997), "Chaotic Behaviour of Gas-Solid Flow in the Riser of a Laboratory-Scale Circulating Fluidized Bed", AIChE J., 43, 1458-1468.

Müuller, U.A., Dacorogna, M.M., Davé, R.D., Pictet, O.V., Olsen, R.B., and Ward, J.R., (1995), "Fractals and Intrinsic TimeA Challenge to Econometricians", $34^{\text {th }}$ In. Conf. of Applied Econometrics Association (AEA), Luxenbourg, 14-15 October, 1995.

Schaffer, W.M., (1985), “Can Nonlinear Dynamics Elucidate Mechanisms in Ecology and Epidermiology?", IMA J. Math. Applied in Medicine \& Biology, 2, 221-252.

Schoulten , J.C., and van den Bleek, C.M., (1998), "Monitoring the Quality of Fluidization Using the Short-term Predictability of Pressure Fluctuations", AIChE J., 44(1), 48-60.

Schoulten, J.C., Takens F., and van den Bleek, C.M., (1994) "Maximum Likelihood Estimation of the Entropy of an Attractor", Physical Review E, 49(1), 126-129.
Soong, C. H., Tuzla, K., and Chen, J.C., (1994), "Identification of Particle Clusters in Circulating Fluidized Bed", in "Circulating Fluidized Bed Technology IV", A. A. Avidan, (Ed.), AIChE, New York, pp. 615620.

Soong, C. H., Tuzla, K., and Chen, J.C., (1995), "Experimental Determination of Cluster Size and Velocity in Circulating Fluidized Bed", in "Fluidization VIII", J-F. Large and C. Laguerie (eds.), Engineering Foundation, New York, pp. 219-227.

van der Stappen M.L.M., Schoulten, J.C., and van den Bleek, C.M., (1993a), "Application of Deterministic Chaos Theory in Understanding the Fluid Dynamic Behavior of Gas-Solids Fluidization”, AIChE Symposium Series, 89 (296), 91-102.

van der Stappen, M.L.M., Schoulten, J.C., and van den Bleek, C.M., (1993b), "Applications of Deterministic Chaos Analysis to Pressure Fluctuations Measurements in a $0.96-\mathrm{m}^{2}$ CFB Riser", CFB IV Conference, pp. 55-60.

Zhang, H., Johnson, P.M., Zhu, J.-X., de Lasa, H.I., and Bergougnou, M.A., (1998). "A Novel Calibration Procedure for an Optical Fiber Concentration Probe", Powder Technol., 100, 260-272. 